\title{
LE HAOUZ DE MARRAKECH ET LE HAUT-ATLAS
}

Si des savants se sont accordés pour considérer le Maroc, l'Algérie et la Tunisie comme une création 'de l'Atlas, allant même jusqu'à proposer l'expression d'Atlasie pour mieux définir en congrès la remarquable unité de ces trois pays, a fortiori le Haouz de Marrakech apparait-il bien comme une authentique création du Haut Atlas, par le jeu des trois phases d'ablation, de transport et de dépôt des matériaux arrachés aux montagnes au cours des temps géologiques,

Mais le sol du Haouz ne serait qu'un don stérile si le Haut Atlas ne lui procurait aussi les ressources d'humidité nécessaires à la vie, par sa prodigieuse barrière de 4.000 mètres où la proximité de l'Atlantique augmente encore les effets de l'altitude.

C'est au Haut Atlas et à lui seul, qui renverse à son niveau la succession des étages bioclimatiques, que l'on doit en effet, sous la latitude du $37^{\circledR}$ parallèle qui marque en Algérie les confins des grands déserts, l'existence de cette zone privilégiée qui suscite de si vastes espoirs de prospérité.

Comment s'exerce au profit du Haouz ce rôle bienfaisant du Haut Atlas, et peut-il être soutenu ?

Telles sont les questions dominantes qu'il n'est pas surprenant qu'un forestier se soit posées tout au long de ce travail.

Or la végétation est bien la manifestation la plus spectaculaire des effets du Haut Atlas et elle constitue l'élément le plus éloquent du milieu physique que nous étudions, puisqu'elle représente à la fois le reflet du climat et du sol.

A ce titre, la végétation forestière s'offre comme le meilleur réactif du milieu, par sa taille qui la rend plus apparente, la sociabilité de ses essences qui confère à chacun de ses représentants la plus haute valeur de témoin, et surtout par les immensités qu'elle recouvrait jadis et que la connaissance des associations végétales permet de retrouver aujourd'hui, en dépit de l'action de l'homme qui a si profon'dément modifié, en même temps que l'étendue de l'emprise forestière, l'état d'équilibre ou ( climax » de la végétation livrée à elle-même.

Examiner attentivement cette végétation telle qu'elle existe aujourd'hui, s'efforcer de saisir et de traduire ses caractères et son 
évolution, n'est-ce pas un bon moyen de rechercher, par référence à d'irrécusables et de si fidèles témoignages, les plus valables réponses aux questions posées?

\section{La véGÉtation dans la plaine du HaOUZ}

Le Haouz est lui-même un terrain d'investigation passionnant pour un forestier.

La brousse à jujubier, réactif de l'étage bioclimatique aride chaud, telle qu'elle est encore bien représentée à l'Est de Tamelelt, s'étendait en effet jadis à peu près sans discontinuité au Nord du Haut Atlas, et, vers l'Ouest, jusqu'à l'arganier comme en témoignent encore, parfaitement visibles le long des routes qui rayonnent autour de Marrakech, quelques arbres remarquables, isolés ou en bouquets miraculeusement épargnés (P. K. 42.500 de la route $\mathrm{N}^{\circ} 7$, P. K. IO5 de la route $\mathrm{N}^{\circ}$ Io) ainsi que de nombreux cimetières musulmans à l'intérieut desquels la végétation s'est trouvée respectée (Marahout (le Sidi Bou Othman).

\section{Les facteurs de dégradation}

Déjà considérablement réduite en surface du fait de l'homme, cette brousse représente aujourd'hui une ultime réserve de parcours pour les animaux, et elle est déjà menacée de destruction par les facteurs de dégradation suivants, qui s'exercent à la fois sur l'essence, sur la végétation associée et sur le sol.

a) Dans toute la plaine, le jujubier est largement utilisé pour l'édification de haies séparatives de toutes sortes, d'enclos à bestiaux et de protections diverses qui consomment chaque année dans chaque douar des tonnes de matières végétales récoltées au plus près sans aucun souci 'des possibilités de l'essence.

Le spectacle familier de la récolte et du transport de ces énormes charges de branchages pour la réfection des "Zérilas 》 suffit à expliquer l'épuisement progressif du jujubier dans les zones où il ne cesse d'être tondu, quand la pratique des extractions des souches et des racines pour la recherche du bois de chauffage ne vient pas encore précipiter sa disparition.

b) Si le parcours permanent des animaux reste l'une des causes principales de la forme buissonnante du jujubier, par « un abroutissement " systématique des jeunes pousses terminales et latérales, ce facteur joue aussi un rôle prépondérant sur la composition, la richesse et la valeur nutritive de la végétation associée et du tapis herbacé en particulier.

La continuité et la surcharge du parcours ont en effet pour résultat d'anéantir brutalement dès les premières pluies des possibilités d'alimentation incomparablement plus importantes qui pourraient être utilisées plus tardivement, en même temps que disparaissent 
progressivement, faute de pouvoir se reproduire, bon nombre de plantes annuelles, recherchées par le bétail et prématurément consommées par ce 'dernier.

Si quelques-unes de ces plantes peuvent échapper au bétail ct fructifier à l'abri des touffes de jujubier considérées comme des centres de dispersion de graines, l'on constate que cet ensemencement limité s'avère très vite insuffisant, et que les parcours surchargés s'appauvrissent et se dégradent rapidement, en ne supportant plus que des espèces épineuses, vénéneuses, ou dédaignées par le bétail.

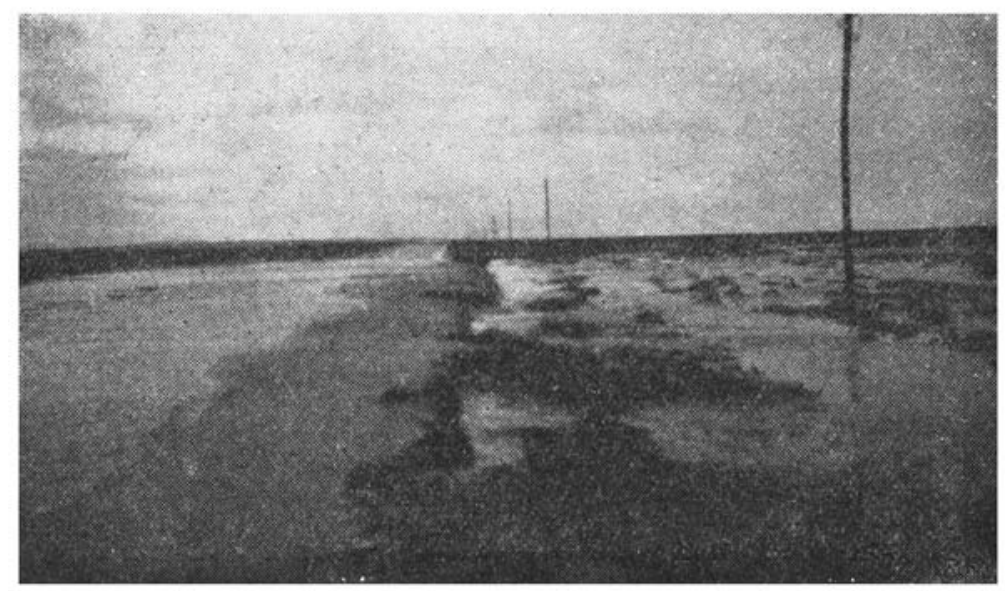

Photo $n^{\circ}$ I: Dégradation des terrains de parcours du Haouz. Les caux de pluie ne sont plus retenues et ruissellent en pure perte sur des glacis imperméables. Le pays s'assèche pragressivement.

c) La brousse de jujubier actuelle, avec ses touffes éparses plus ou moins abrouties, $\cdot$ protège incomparablement moins bien le sol qu'un véritable peuplement de sujets plus élevés, et il serait vain de rechercher unc trace d'humus quelconque entre ces touffes, sur un sol directement exposé aux agents atmosphériques, systématiquement nettoyé de tout tapis végétal, et continuellement piétiné par le passage des animaux.

\section{Imperméabilité de la couche superficielle}

Bien que les dépôts alluvionnaires du Haouz puissent atteindre une grande profondeur, de tels sols insuffisamment protégés et piétinés perdent très facilement leur perméabilité par la formation d'une couche superficielle plombée, dont les inconvénients sont en- 
core aggravés, dès que le sel apparaît, par le phénomène de " glaçage " provoqué par l'argile sodique, qui rend les sols compacts, imperméables et asphyxiants.

La démonstration de l'imperméabilité de ces sols, qui doit être considérée comme un signe de dégradation avancée, nous est fournie à chaque pluie dans tous les points bas du Haouz, qui sont le théâtre de phénomènes de ruissellement absolument inattendus dans ce pays plat, et qui sont provoqués par l'écoulement 'des eaux de surface qui devraient être beaucoup mieux retenues et utilisées par ces terrains à faible pente, s'ils se trouvaient en meilleur état (Photo $\left.\mathrm{n}^{0} \mathrm{I}\right)$.

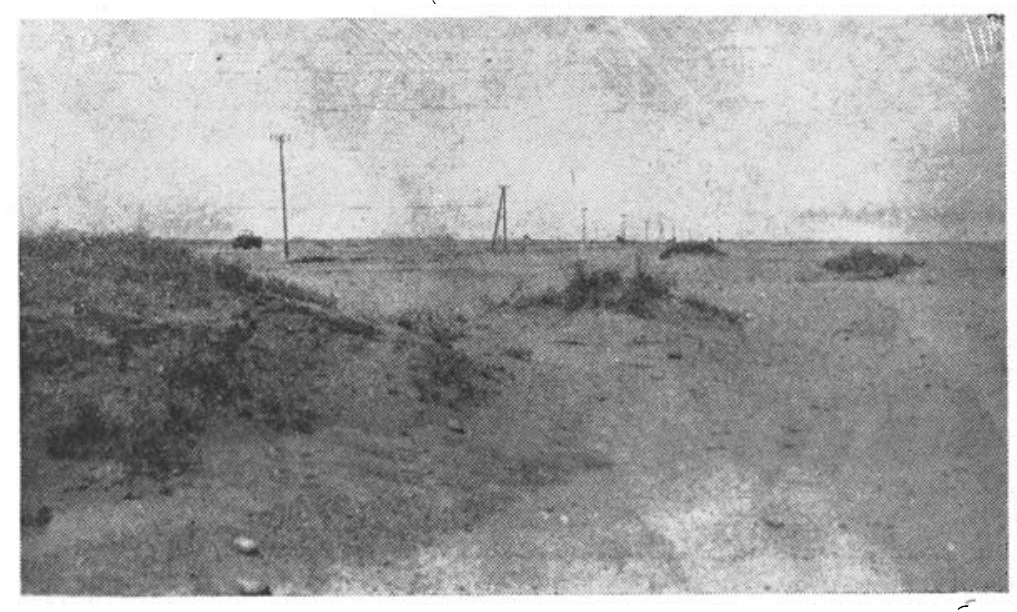

Photo $n^{\circ}$ 2: D'égradation des terrains de parcours du Haouz. L'érosion éolienne s'exerce pendant les longs mois de sécheresse.

Enfin, ces sols privés progressivement de leur armature végétale, dépourvus d'humus et desséchés, deviennent la proie d'une érosion éolienne particulièrement grave que rien ne peut arrêter (Photo $\left.n^{\circ} 2\right)$.

\section{Premières conclusions}

Quelles sont donc les premières conclusions que cet examen nous autorise à formuler ?

Comme il existe dans le Haouz plus de terres propres à l'irrigation que d'eau pour les irriguer, on en déduit qu'en dehors de l'emprise des périmètres actuels ou projetés, il faut à la fois dans toute la partie restant du Haouz, conserver dans le sol les eaux pluviales qui ruissellent en pure perte, maintenir, restaurer et enrichir les 
terrains de parcours en voie de dégradation qui ne pourront faire l'objet d'aucune utilisation, développer les plantations forestières et fruitières partout où elles sont possibles dans le but:

- de satisfaire rapidement les besoins en bois de feu et de service d'une population souvent réduite à dessoucher le jujubier, et de lui procurer éventuellement, par l'arboriculture fruitière un appoint alimentaire ou économique appréciable.

- de créer des bosquets-abris indispensables aux animaux dans les zones de parcours.

- de faire obstacle à la violence des vents pour lutter contre l'érosion éolienne.

- de maintenir au moins, et d'améliorer sans doute par des massifs boisés, les caractéristiques déjà sévères de l'étage bioclimatique aride chaud du Haouz, car. il ne faut pas se dissimuler que parmi les trois éléments constitutifs et inséparables du milieu physique, la dégradation de la végétation et celle du sol sur des vastes étendues entraînent inévitablement une dégradation du climat, qui tendrait à devenir encore plus sec et encore plus chaud. Ces considérations ne restent pas des vues de l'esprit.

Unité des différents problèmes physiques, économiques et sociaux dans la plaine du Haouz et le Haut Atlas

L'Inspection des Eaux et Forêts de Marrakech a saisi l'unité des problèmes physiques, économiques et sociaux qui consacrent l'indissociable parenté du Haouz et du Haut Atlas, et senti la nécessité d'appliquer à la plaine, en les adaptant, les mesures générales de sauvegarde et de protection qui sont bien familières aux forestiers.

\section{Lutte contre le ruissellement}

C'est ainsi que les premiers travaux de lutte contre le ruissellement par la méthode du barrage des pentes et des ravins ont été commencés en 1946 dans le périmètre de reboisement des Djebilet (dont il sera plus anplement question dans le chapitre suivant consacré à la montagne), que des expériences d'amélioration pastorale sont poursuivies depuis 1948 dans des terres de parcours collectives à jujubier de l'annexe de Chichaoua et des circonscriptions d'Amizmiz, des Rehamna et des Srarhna, pour un total de I.O00, hectares et que 150 hectares de reboisement massif d'eucalyptus de rapport ont été réalisés depuis 1949 sur des terres collectives des Srarhna.

Quel est l'avenir de ces entreprises?

\section{Avenir des essais poursuivis}

Le développement de la lutte contre le ruissellement pour la conservation du sol et de l'eau devant être étudié plus loin, disons de 
suite que le premier programme de reboisement des terres collectives des Srarhna porte sur plus de I.500 ha avec des possibilités d'extension susceptibles de décupler cette surface en bordure du futur périmètre d'irrigation.

Quant à l'amélioration des terres collectives de parcours dégradées du Haouz, dans lesquelles le reboisement par bosquets-abris et par brise-vents doit avoir sa part, elle semble ne devoir connaître pour limite que celle de ces terres collectives elles-mêmes, lorsque les résultats obtenus dans les parcelles d'expérience en cours, pourtant déjà si démonstratifs, se seront définitivement imposées à l'attention de tous.

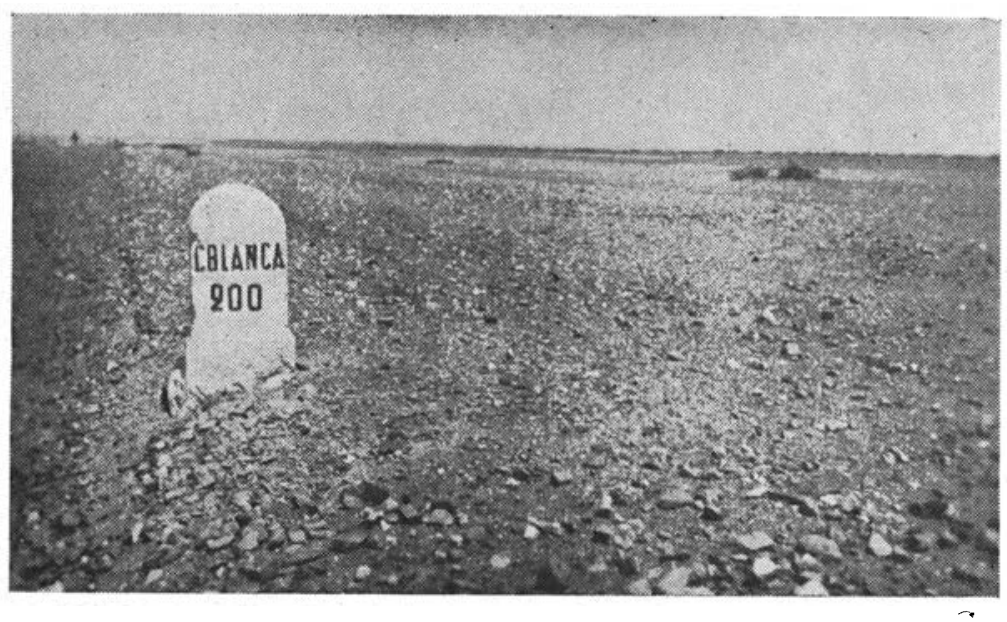

Photo $n^{\circ}$ 3: Restauration pastorale à Bounaga. Témoin à l'est de la route de Marrakech à Casablanca.

\section{Dispositions législatives}

Des dispositions législatives récentes permettent en effet de soumettre au régime forestier les terres collectives de parcours à améliorer par l'administration des Eaux et Forêts, et les moyens techniques pour y parvenir sont expérimentés de la façon la plus complèté, et la plus nuancée dans la parcelle de $5_{50}$ ha de Bounaga ( $P$. K. 40 de la route de Marrakech à Casablanca) (Photos $n^{\circ} 3$ et 4 ).

On ne doute pas qu'un aménagement pastoral minimum, consistant à substituer un parcours circulaire et réglementé au parcours linéaire actuel qui stérilise tout sur son passage, ne soit très prochainement institué dans l'annexe de Chemaia sur plusieurs milliers d'hectares, et que ce mouvement ne gagne ensuite le Haouz tout entier, à mesure que tomberont une à une les réticences d'un milieu humain encore peu accessible au concept de l'intérêt général. 
Comme il serait illogique de créer à grands frais de nouvelles ressources tout en laissant à côté se ruiner d'autres richesses naturelles providentielles pour cette latitude, c'est à un ensemble d'entreprises coordonnées et cohérentes que le forestier songe d'abord pour l'équilibre durable du' Haouz, avant de tourner maintenant ses regards vers les forêts du Haut-Atlas.

\section{Le Haut Atras}

Le secteur du Haut-Atlas qui domine le Haouz fait partie de la $\mathrm{X}^{\mathbf{e}}$ région forestière du Grand-Atlas, telle qu'elle est définie

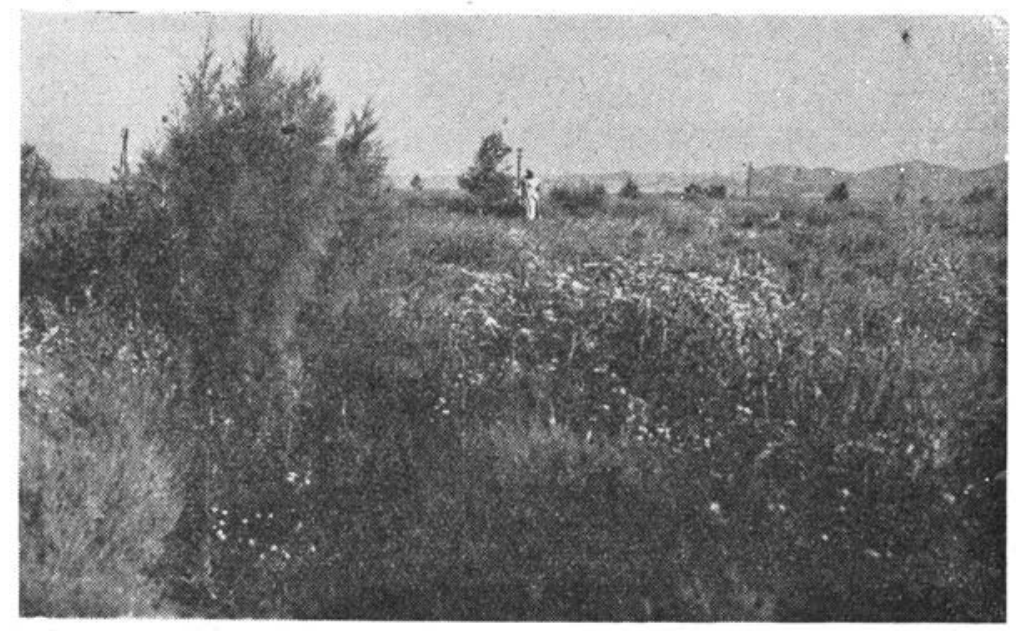

Photo $n^{\circ}$ 4: Restauration pastorale à Bounaga. Résultats après moins de 3 ans de protection à l'ouest de la route.

au tome premier de " l'Economie Forestière Nord-Africaine » de M. Boudy, ancien directeur des Eaux, et Forêts du Maroc, avec une superficie boisée de 600.000 ha et un taux de boisement de 28 , I.

La chaîne centrale est elle-même constituée de deux parties géologiquement distinctes que sépare en gros la vallée de la Tessaout avec le massif de terrains anciens et éruptifs à l'Ouest et les puissantes formations jurassiques à l'Est, ce qui explique les physionomies si différentes de deux sommets d'aftitude comparable comme le Toubkal (4.165 m) et. l'Irhil Mgoun (4.070 m).

La succession altitudinale des étages bioclimatiques semi-aride', sub-humide (tempéré), humide et de haute montagne est ici modifiée par l'installation d'un étage relativement sec (semi-aride froid) 
qui se place entre les étages humide et de haute montagne, au détriment du premier dont il diminue l'étendue.

Les essences forestières sont par ordre d'importance le chêne vert, véritablement caractéristique du Haut-Atlas, qui occupe les deux tiers de la surface boisée de la région, les genèvriers oxycèdres et de Phénicie, le Thuya, le pin d'Alep, le genèvrier Thurifère et le cyprès de l'Atlas, de la haute vallée du N'Fis.

La limite altitudinale de la végétation forestière ne dépasse pas $3.009 \mathrm{~m}$ avec le genèvrier thurifère et il est important de noter le développement que prend ici l'étage bioclimatique de haute montàgne, qui apparait vers $2.800 \mathrm{~m}$ et qui, pour être essentiellement asylvatique, ne doit cependant pas échapper à l'attention de ceux qui se préoccupent de l'équilibre physique de la montagne.

Or, le taux de boisement de $28, \mathrm{I}$ de la $\mathrm{X}^{\boldsymbol{\theta}}$ région forestière du Gran'd Atlas ne représente qu'un résidu et, si le Maroc a perdu près de 5 , millions d'hectares de forêts détruites depuis la période historique, il est important de savoir que les surfaces forestières disparues dans cette $\mathrm{X}^{\mathrm{e}}$ région pendant le même temps sont estimées à 600.000 ha, c'est-à-dire qu'il ne nous reste plus aujourd'hui que la moitié de la surface des forêts d'autrefois.

Le chêne vert, en particulier, que l'gn ne rencontre plus guère avant $\mathrm{I} .200 \mathrm{~m}$ 'd'altitude aujourd'hui, occupait jadis les premiers contreforts de la montagne à partir de $600 \mathrm{~m}$ alors qu'il ne reste plus maintenant sur le ( Dir » quel les derniers vestiges de l'association végétale du " climax ) disparu, sous la forme de ces vastes nappes de palmier nain que des exploitations abusives menacent encore de faire disparaitre sous nos veux.

\section{Causes de la déforestation}

Les causes humaines de déforestation sont connues: défrichements, incendies, abus d'exploitation et de pâturages, écorçage des arbres sur pied ou des racines pour la récolte du tanin.

La fondation de la ville de Marrakech, il y a 900 ans n'a fait qu'intensifier des destructions de bordure qui se sont exercées dc tous temps partout où la vie humaine s'était installée dans les vallées, avec une puissance variable, reflétant les vicissitudes mêmes de ces populations, et la récente pacification française, par ses conséquences sur l'accroissement démographique et l'élévation des niveaux de vie, a singulièrement augmenté la menace que fait peser l'érosion humaine aussi bien sur les massifs boisés que sur les parcours asylvatiques de haute montagne.

\section{- La politique forestière suivie dans le Haut-Atlas depuis I9I8}

Le milieu humain qui a si considérablement diminué dans le Haut-Atlas la surface des forêts et la qualité de ce qu'il en reste 
nous apparaît donc absolument inséparable du milieu physique et cela domine toute la politique forestière à Marrakech depuis r9r8, date de la création de la première circonscription forestière, et plus efficacement depuis I922 qui marque le début de l'implantation du service dans la montagne par la construction du premier poste forestier d'Agaiouar.

Dix ans après, et sans doute après beaucoup d'autres forestiers qui ont servi ou servent encore en Afrique du Nord, j'ai moi-même cléfini comme il suit l'essentiel de cette politique dans des lettres transmises en I933 et les années suivantes aux autorités locales intéressées, relatives aux défrichements par le feu que je signalais sur des pentes abruptes:

“ Ces boisements, directement ou indirectement, peuvent être " considérés comme une source de richesse des plus importantes " pour la population en même temps qu'ils constituent l'armature " la plus solide et la parure la plus belle de ces montagnes.

“ L'action préservatrice du Service Forestier pourrait peut-être (" s'exercer utilement dans cette région montagneuse où il est à " craindre que l'insouciance des indigènes ne fasse prématurément " disparaitre, sur les pentes accentuées, les peuplements qui main(" tiennent aujourd'hui la terre végétale et qui régularisent le débit (" des eaux... )

Et plus loin:

“ S’il est entendu que la forêt de montagne, déjà considérée com" me réserve de pâturage, doit largement admettre en son sein la “ charrue de l'usager dans les plateaux à vocation agricole durable (" et les versants peu inclinés, il serait par contre souhaitable de voir (" disparaitre les mises à feu sur les pentes abruptes, ce système de " culture empirique s'étant révélé comme le plus sûr moyen de (" cétruire le boisement et de livrer le sol à l'érosion. )

Et d'autre part:

“ Il doit exister ici, comme dans toute économie montagnarde (" bien comprise, un juste partage des terres entre l'agriculture, les ( cultures irriguées, l'arboriculture, l'élevage et la forêt considérée (" conme la seule réserve de pâturage, la seule armature des ver(" sants, et le seul élément régulateur des eaux pluviales et couran" tes. Cette harmonie est malheureusement rompue depuis longtemps " dans ce sècteur, dont les terres des cultures sont fatalement limi" tées comme partout, et dont l'effort d'extension des labours n'a " d'autres résultats que le pillage de la forêt.

“ Ft la solution de ce problème ne réside pas à mon sens dans " la déforestation systématique de la Tribu, mais bien dans les " améliorations agricoles et pastorales de ce pays où tout reste à « faire. 
“ Il n'est pas soutenable que les meilleurs terrains de montagne ne " pourraient pas être mieux cultivés par les Berbères lorsque l'his" toire nous apprend que les Anciens connaissaient et pratiquaient " déjà le système de la jachère cultivée et des labours préparatoires. "C'est donc vers une agriculture plus soigneuse, plus laborieuse “ e plus raisonnée des bons terrains qu’il faut orienter aujourd'hui " les indigènes, en leur faisant comprendre l'impérieuse nécessité " d'accroître le rendement de propriétés 'dont il n'est plus possible " d'augmenter la surface. ")

\section{Autres conclusions}

Et enfin, comme conclusion d'un rapport d'ensemble :

“ Le but élevé que s'est tracé le Service Forestier dans ces “ régions a été maintes fois précisé, il consiste avant tout à restau" rer une montagne délabrée, à rétablir son équilibre compromis, " à maintenir, améliorer et augmenter ses ressources naturelles, à " la renclre plus riche, plus prospère, plus accueillante, plus hospi" talière, plus confortable et plus riante pour ceux qui l'habitent, " en même temps qu'une régularisation meilleure des eaux cou" rantes dans les bassins condensateurs assurera dans les plaines " agricoles un débit plus constant 'des rivières et une protection " plus efficace contre les inondations dévastatrices.

“ Ne pas entendre les cris d'alarme que nous poussons, hési" ter devant les difficultés passagères d'application des mesures " de protection les plus élémentaires et les plus efficaces des mas“ sifs boisés, différer plus longtemps la solution d'un problème " qui se complique de plus en plus chaque année, c'est ne rien " changer à l'insouciante anarchie de ceux que nous assurons de " notre protection, c'est sacrifier de la manière la plus dangereuse “ l'avenir au présent et compromettre gravement la politique de (( demain. )

L'on saisit par cette conclusion quelles ont été et quelles sont encore les préoccupations majeures des forestiers de Marrakech auxquels les rapports étroits du Haut-Atlas et du Haouz n'ont jamais échappé.

Mais le milieu humain est en jeu et il n'a pas fallu moins de 30 ans de patients efforts, avec toute la souplesse, la diplomatie et la persuasion qu'ils représentent, pour assurer sur l'ensemble du versant Nord du Haut-Atlas qui domine le Haouz la protection essentielle des massifs boisés contre les défrichements, les abus d'exploitation et les incendies, combattus maintenant avec la participation dos rizorains dans presque tous les cas. 
La sauvegarde des forêts se montre-t-elle aujourd'hui suffisante?

L’équilibre physique de la montagne se trouve-t-il durablement assuré pour elle-même et pour les bienfaits qu'elle dispense à la plaine?

Certainement non, et de nombreuses tâches s'imposent encore, ou restent à accomplir.

\section{LES TACIIES RESTANT A ACCOMPLIR}

\section{Régénération et reboiscment forestiers}

a) Il a été signalé que l'érosion humaine s'exerçait non seulement en bordure de forêt, mais aussi dans sa masse, en sorte que la végétation du " climax ) a généralement subi de graves altérations en se transformant progressivement en peuplements de plus en plus dégradés, dont le maquis, la broussaille et les chamœphytes sont les différents stades. La sylviculture s'attache à favoriser la régénération et le cléveloppement des essences principales les plus précieuses, en même temps que les travaux de repeuplement et de reboisement enrichissent peu à peu les landes pelées qui attristent encore trop souvent de leurs vides nos périmètres forestiers.

\section{Pâturage}

b) I.e pâturage est sans conteste le droit d'usage le plus important qui grève les forêts du Haut-Atlas mais son exercice déréglé ou abusif, surtout par les caprins, est aussi l'un des puissants factcurs de destruction de l'état boisé.

Tout le problème consiste à déterminer dans quelle mesure la forêt peut admettre sans péril les troupeaux des usagers et le recensement de ce cheptel est actuellement poursuivi dans toutes les tribus de montagne ainsi que l'a demandé lui-même Son Excellence le Grand Vizir Si E1 Mokhri, lors de la réunion en mai I944, de la Commission de 1'Economie marocaine où fut discutée et adoptée la question du régime sylvo-pastoral de la forêt marocaine (Arrêté viziriel du i6 avril i946).

c) La poursuite de l'œuvre exige encore d'autres efforts jusque clans l'étendue de l'étage bioclimatique asylvatique de haute montagne dont nous avons fait remarquer le développement considérable dans le Haut-Atlas. Certes le pâturage s'exerce sur la chaîne tout entière et la zone essentielle en est le domaine forestier, mais le déplacement des troupeaux devient nécessaire et se complique singulièrement pour les habitants de la haute montagne avec le mouvement de descente vers les plaines, imposé par la neige en hiver, et la remontée vers les alpages en été, selon des règles traditịnnelles, 
sinon techniques, qui varient de l'azib particulier appartenant au village, à l'alpage collectif dépendant de plusieurs tribus.

$\mathrm{Si}$ la paix règne maintenant jusqu'au sommet du Haut-Atlas dans ces précieux alpages, âprement disputés autrefois par les armes, il semble bien qu'une discipline soit nécessaire pour la maintenir et que la protection du Saint local ne suffise plus aujourd'hui pour préserver ces richesses de l'inévitable destruction dont elles sont menacées.

Le montagnard qui n'a aucune confiance dans la monnaie et qui ne peut guère investir sur place son argent dans sa vallée, où la terre est rare, considère en effet le troupeau comme une banque mobile très pratique, et il en augmente indéfiniment le nombre dans les années de prospérité, malgré le risque que lui font périodiquement courir les années de sécheresse ou les épizooties.

L'appauvrissement corrélatif de la flore de haute montagne, recherchée par le bétail, peut être déjà constaté sur bien des alpages surchargés, et il est malheureusement trop certain que la dégradation de la végétation provoque aussi celle du sol qui devient immédiatement la proie de l'érosion.

A la base même de l'équilibre physique et démographique de la haute montagne, une réglementation technique de l'exercice de ce pâturage s'impose absolument, portant sur les charges d'animaux admissibles, sur la mise en repos périodique des terrains et sur l'exécution de travaux d'amélioration.

L'on ne cloute pas des difficultés matérielles que représente une semblable tâche dans les alpages ni des qualités exceptionnelles qu'elle exigera d'un personnel technique nécessairement lié au mouvement des troupeaux. Encore ces difficultés d'exécution seront-elles plus faciles à résoudre, pcut-être, que celles de faire admettre le principe même de la protection des pâturages de haute montagne, que leur situation juridique semble pourtant bien classer pour la plupart dans la catégorie des biens inaliénables revenant de droit au Maghzen tels qu'ils sont définis par le dahir du 7 juillet I9I4 sur la propriété immobilière.

\section{Défense et restaunation des sols de montagne}

Enfin, des travaux de défense et de restauration des sols pour la conservation de la terre et de l'eau (D.R.S.) deviennent nécessaires depuis le Haouz jusqu'aux sommets du Haut-Atlas et l'on espère que la généralisation de ces entreprises marquera dans quelques décades le couronnement de l'œuvre des forestiers du Sud Marocain qui consacrent leurs efforts au maintien de l'équilibre physique et clémographique de la plaine et de la montagne.

L'érosion du sol par l'eau de ruissellement est évidemment la forme du mal la plus grave et la plus répandue contre laquelle s'im- 


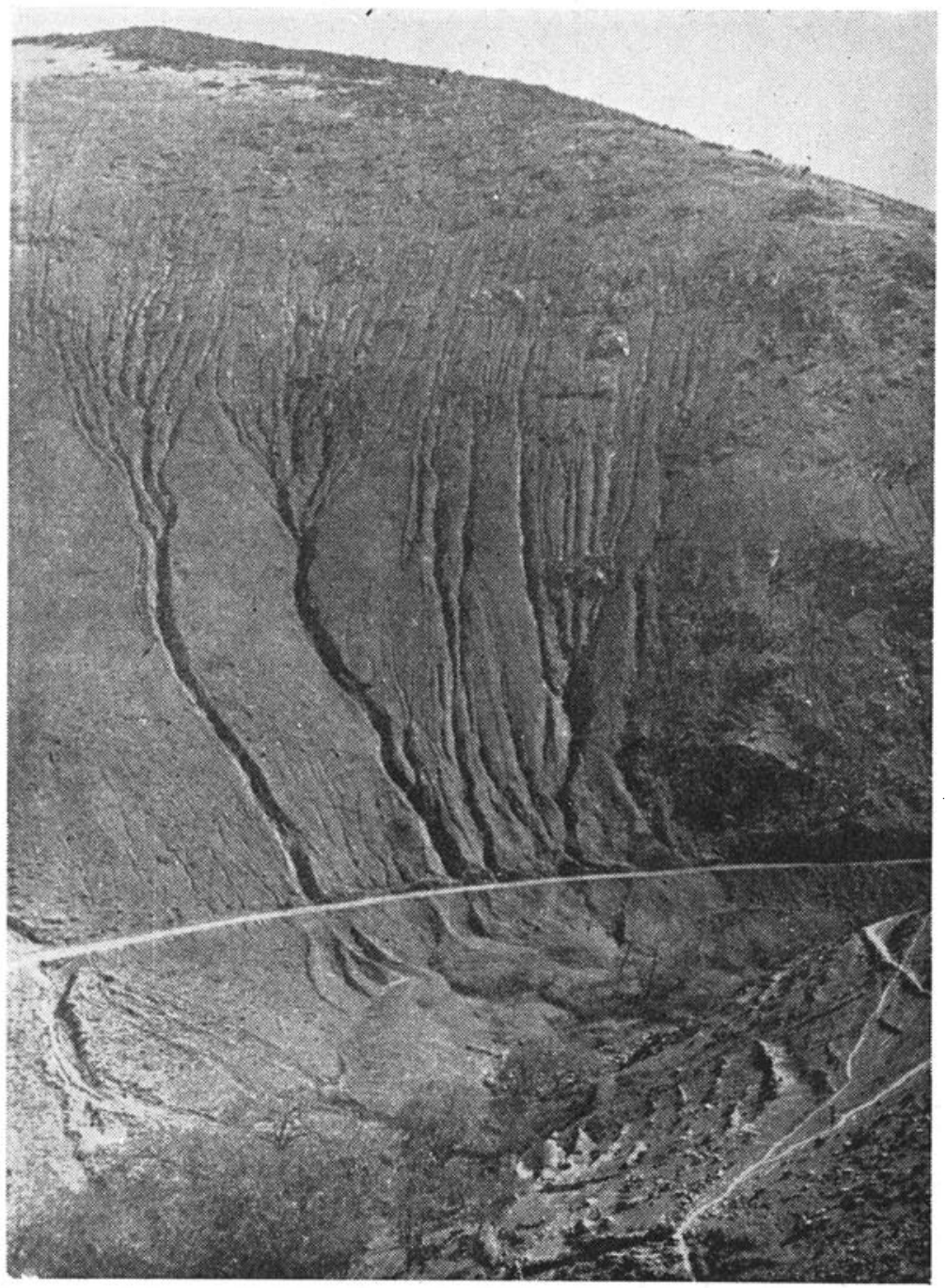

Photo $n^{\circ}$ 5: Exemple d'érosion pluviale à son origine dans le Haut Atlas vers $1.800 \mathrm{~m}$ d'altitude 
pose la iutte, les effets les plus saisissants du ruissellement nous étant offerts par les phénomènes torrentiels que nous observons sous nos yeux dans les montagnes dénudées, principalement après la longue sécheresse de l'été, pendant laquelle le sol est plus ou moins décomposé. Nous assistons alorś à un véritable décapage 'des croupes et des versants par les eaux qui se précipitent dans les ravins avec les matériaux qu'elles entrainent pour former aussitôt d'indomptables torrents qui arrachent leurs berges, font écrouler des versants et finissent par ensevelir sous leurs laves de larges étendues.

Tous les habitants de Marrakech ont encore présent en leur mémoirc le souvenir des affreuses dévastations qui attristèrent le Haouz ct la ville en mai I949 et plus récemment à l'automne dernier, qui se chiffrent par des vies humaines et plusieurs containes de millions de ldégâts matériels sans compter les millions de mètres: cubes d'cau et do bonne terre végétale entrainés cn pure perte vers l'Océan (Photo $\mathrm{n}^{\circ}$ 5).

\section{Témoignage algérien}

L’importance du décapage, avec la stérilisation et l'assèchement des versants qui en résultent, et dont on devine les funestes conséquences économiques et sociales, s'accélère à une cadence incroyable clont témoignent les observations relevées dans les cuvettes des barrages d'Algérie.

Le barrage des “Cheurfa ) (St Denis du Sig - Oran) est tombé d'une capacité 'de 16 millions de mètres cubes vers I900, à 5 millions et s'envase à raison de 400.000 mètres cubes par an: c'est un agonisant, nous dit M. Dumont, expert au Commissariat du plan de modernisation et d'équipement.

Le barrage de l'oued Fodda (Moyen Chellif) de 225 millions de mètres cubes de capacité pour un bassin de versant de 76.000 hectares, achevé en I932, a dès I948, 40 millions de mètres cubes stérilisés par la vase, et il se comble à raison de 4 millions de mètres çubes par an, cadence qui tend à s'accélérer, et qui correspond aujourd'hui à un décapage moyen de près de $8 \mathrm{~mm}$ des terres nues ou non protégées du bassin qui couvrent $66 \%$ de sa surface totale.

\section{Les remèdes}

In premier remède qui s’impose pour maintenir la solidité de la montagne est de conserver d'abord partout où elle existe la moindre végétation et principalement la végétation forestière qui, avec sa tamure, son humus et son enracinement est évidemment le meilleur élément de conservation du sol et de l'eau par le mécanisme qui est ainsi résumé dans l'introduction d'un ouvrage technique sur la question : 
"Supposons toutes les rampes montagneuses garnies de forêts " ct engazonnées, il n'y aurait plus, à proprement parler, de tor" rents, car ces vastes espaces composeraient comme une immense " éponge retenant les eaux pluviales ou les neiges et distillant goutte " à goutte le liquide absorbé. Les glaciers seuls et les névés cons" titueraient les cours d'eau normaux, mais les glaciers comme les " névés jaugent l'écoulement de la fonte avec une grande régu" larité.

" Si les cours d'eau normaux ne rencontraient sur leur parcours " jusquı'à la plaine que le résultat de cette distillation lente des " terrains couverts de végétation et non les émissions brusques et " terribles des torrents grossis par les pluies, ils gonfleraient pai" siblement et jamais au point d'envahir en un jour d'énormes " espaces pour laisser bientôt après la sécheresse s'étendre sur ces ( mêmes surfaces. )

\section{Leur efficacité}

Iorsqu'une éponge est saturée d'eau, il devient évidemment impossible de lui en faire absorber de nouveau, et l'on ne soutient pas que la végétation forestière puisse supprimer toutes les crues des rivières, ni les inondations consécutives à des pluies diluviennes, mais au moins cet excès d'eau s'écoulera-t-il au bas de chaque versant par la masse même de l'éponge sans ruissellement ni entraînement de matériaux, ce qui est le point capital à retenir.

Malheureusement, sous les climats nord-africains la cléforestation et le reboisement ne sont pas toujours reversibles, et l'on ne peut évidemment songer à reboiser les quelque 600.000 hectares de forêts disparus dans 1a. Xe région du Grand Atlas depuis la période historique. euvre techniquement et socialement impossible, mais il n'est pas cle petits moyens qui ne puissent être mis en œuvre sur ces mêmes étendues pour faire obstacle à l'eau 'de ruissellement. pour chercher à la retenir avec le sol là où elle tombe, pour l'empêcher l'accélérer sa vitesse, et pour la conduire sans dommage dans le prochain ravin.

\section{Lutte contre la goutte d'eau}

La lutte contre la goutte d'eau doit logiquement intervenir dès l'instant que celle-ci touche le sol et elle doit être organisée et généralisée dans le Haouz comme dans le Haut-Atlas. Le Service de la D.R.S. créé en 1949 lui consacre tous ses efforts.

Les premières possibilités d'intervention dans l'étage bioclimatique de haute montagne correspondent d'abord au programme de réglementation technique du pâturage tel qu’il a été précédemment exposé, car il n'est pas de moyen de lutte plus efficace que de reconstituer sur des vastes surfaces le tapis végétal 'des alpages menacé de disparition, à des altitudes supérieures à 3.000 mètres où les précipitations affectent sans doute une forme neigeuse moińs 
dangereuse pour l'érosion, mais qui sont aussi le théâtre de violents orages dont les effets sont parfois foudroyants, quoique généralement localisés.

\section{Barrages des pentes}

Partout ailleurs, où la vie humaine s'est accrochée au sol par la culture, le Service de la D.R.S. s'efforce de mettre en œuvre l'originalité de ses méthodes qui consistent à intéresser les propriétaires eux-mêmes à la conservation, à la défense et la restauration de leurs terrains par les procédés les plus simples et les moins onéreux (labour selon les courbes du niveau, cordons de pierres horizontaux en chicane, haies vives horizontales, cultures par bandes, plantations sur banquettes de niveau, fossés horizontaux, petits barrages des ravins, etc...).

Tous les terrains de culture peuvent déjà bénéficier de ces moyens élémentaires de conservation du sol et de l'eau, et l'on fonde de gran'ds espoirs, en particulier sur l'application généralisée de la méthode du barrage des pentes par des banquettes de niveau avec bourrelets dont l'équidistance et les dimensions peuvent être facilement calculées dans chaque cas.

Ce système permet de récupérer et de valoriser rapidement des terrains déjà dégra'dés et même ruinés sur lesquels le ruissellement est rigoureusement arrêté. L'eau qui est obligée de s'infiltrer dans un sol qui n'est plus dévoré par l'érosion profite immédiatement à la végétation. dont la rapidité de reconstitution est absolument surprenante et permet de gagner à la culture, par des labours horizontaux entre les banquettes. d'importantes surfaces abandonnées ì elles-mêmes.

\section{Plantations d'arbres fruitiers}

Enfin, des plantations d'arbres fruitiers, adaptés au climat et au terrain sont à recommander dans le bourrelet des banquettes elles-mêmes où l'arbre bénéficie nettement de la fertilisation rapiḍe d'un sol ameubli et humide appelé précisément " effet de bourrelet ").

Ainsi que l'a fait remarquer M. Boudy dans son ouvrage déjà cité, " des travaux de cet ordre, avant déjà donné les résultats les plus encourageants, avaient été entrepris avant la lettre dans la chaine des Djebilet, au Nord de Marrakech, grâce à une initiative audacieuse et heureuse de l'Inspection de Marrakech ».

Ces travaux, commencés en 1946, couvrent aujourd'hui plus de I50 ha, mais l'intérêt de ces expériences, que M. Lowdermilk a signalé lui aussi en 1948, dépasse largement le cadre de la technique, car on s'attache à créer dans ce périmètre une économie stable pauvant servị d'exemple pour la chaine tout entière et basée sur la 
culture rationnelle des céréales, le parcours réglementé des animaux et l'arboriculture (avec ressources complémentaires des figuiers de Barbarie).

Depuis 1946, d'autres secteurs de démonstration de lutte contre le ruissellement et de mise en valeur de terrains dégradés ont été créés dans le Haut-Atlas dans le domaine forestier, dans des terres collectives (Imintanout), ou dans des terrains particuliers (SidiRahal), sur une superficic qui dépasse aujourd'hui un millier d'hectares. Non seulement les résultats immédiats escomptés ont été partout couronnés de succès, mais on ne saurait passer sous silence, à l'actif de ces travaux, l'augmentation du débit du puits de la maison cantonnière des C.F.M. des Djebilet, qui avait complètement tari, ainsi que l'extinction complète des deux torrents issus du Djebel Ourgouz qui domine Imintanout.

Si, dans la législation de la D.R.S. qui est attendue (I), des travaux obligatoires seront certainement prévus dans les cas où l'érosion menace directement des agglomérations, des ouvrages publics ou d'utilité publique, ou des régions agrícoles, on espère aussi que les cultivateurs et éleveurs marocains saisiront un jour leur intérêt d'exécuter, eux-mêmes, sur leurs terrains par exemple et avec l'aide et les encouragements de l'Etat, les travaux nécessaires à la conservation du sol et ide l'eau.

Ainsi, le milieu humain solidairement responsable de la dégradation des plaines et des montagnes; s'associera-t-il étroitement, par un juste renversement de la situation, à l'œuvre immense de défense et de restauration dont le forestier Demontzey a pu dire: " Je ne sais pas de plus noble mission que celle d'aider la nature à reconstituer dans nos montagnes - et j'ajoute aussi dans nos plaines - l'ordre qu'elle avait si bien établi et que seuls l'imprévoyance et l'égoïsme de l'homme ont changé en un véritable chaos ».

Dans cette étude, qui conduit des portes de Marrakech aux sommets les plus élevés du Haut-Atlas, le milieu humain est constanment apparu comme un facteur d'une rare puissance ayant déjà profondément modifié le jeu des forces naturelles, avec des conséquences qui rendent encore plus évidents les inséparables rapports entre la plaine et la montagne.

Le rôle de château d'eau du Haut-Atlas est évidemment primordial et l'on peut avancer que l'équilibre physique de la montagne conditionne à ce titre dans une large mesure l'avenir économique et

(I) N.D.L.R. - Le Dahir et l'arrêté viziriel des 20 et 24 mars I95I sur la défense et la restauration des sols au Maroc ont été publiés après la rédaction de cette étude. 
social du Haouz, tel que la création des futurs périmètres d'irrigation dans ses meilleures terres permet de l'imaginer.

Or, nous avons énuméré les tâches immenses qui restent encore à accomplir pendant plusieurs décades, pour lutter contre les dégradations les plus désastreuses, pour maintenir, défendre et restaurer, tout en tenant compte de l'accroissement démographique et des besoins accrus des populations.

Sans aller jusqu'à proposer la création d'un « Haouz-Haut-Atlas Authority ) pour le règlement coordonné de tous les problèmes dans leur ordre d'urgence, on n'hésitera pas à déclarer que les bienfaits du Haut-Atlas pour le Haouz ne se maintiendront pas sans que d'importants moyens soient mis à la disposition des techniciens responsables et sans qu'une inébranlable volonté d'action n'anime. sincèrement les autorités politiques à tous les échelons, principalement dans cette lutte sans merci que chacun doit soutenir pour la conservation du sol et de l'eau.

Un soir, il y a près de 20 ans, l'histoire suivante m'a été racontée dans un petit village de la Haute-Tessaout à l'heure où, après les fatigues de l'étape, l'arôme délicat du thé à la menthe délie les muscles et les langues:

Un puissant chef de la montagne avait offert une si grandiose réception dans sa casbah que l'appétit des invités ne put venir à bout des victuailles dont les restes furent jetés dans la rivière et entraînés par le flot jusque clans l'Oum er Rbia où des pêcheurs découvrirent dans leurs filets bon nombre de poissons morts d'indigestion de couscous, comme ils s'en aperçurent facilement en ouvrant le ventre des victimes. Le fait fut aussitôt rapporté au Sultan qui châtia sévèrement, en détruisant tous ses biens, le mauvais Caid qui avait gaspillé des aliments en les jetant dans la rivière.

Je pense que la terre du Haut-Atlas est encore plus précieuse aujourd'hui que n'étaient les victuailles au temps de cette histoire et que les moyens modernes d'analyse scientifique des eaux devraient permettre de faire repérer et châtier comme ils le méritent ceux qui ont encore la coupable imprudence de laisser échapper dans les rivières la terre précieuse qui les nourrit.

Marrakech, le 20 février I95I.

Plateau.

Inspecteur des Eaux et Forêts, Adjoint au Chef du Service de la Défense et de la Restauration des sols au Maroc.

Service de la mise en valeur: Monographie du Haouz de Marrakech et Bulletin économique et social du Maroc ( $3^{\circ}$ trimestre I95I) 